\title{
A review of $\mathrm{H}_{2} \mathrm{CO} 6 \mathrm{~cm}$ masers in the Galaxy
}

\author{
E. Araya ${ }^{1,2}$, P. Hofner ${ }^{1,2}$ and W. M. Goss ${ }^{2}$ \\ ${ }^{1}$ National Radio Astronomy Observatory, P.O. Box 0, Socorro, NM 87801, USA \\ ${ }^{2}$ New Mexico Institute of Mining and Technology, Physics Department, 801 Leroy Place, \\ Socorro, NM 87801, USA \\ email: earaya@nrao.edu
}

\begin{abstract}
We present a review of the field of formaldehyde $\left(\mathrm{H}_{2} \mathrm{CO}\right) 6 \mathrm{~cm}$ masers in the Galaxy. Previous to our ongoing work, $\mathrm{H}_{2} \mathrm{CO} 6 \mathrm{~cm}$ masers had been detected in the Galaxy only toward three regions: NGC 7538 IRS1, Sgr B2, and G29.96-0.02. Current efforts by our group using the Very Large Array, Arecibo, and the Green Bank Telescope have resulted in the detection of four new $\mathrm{H}_{2} \mathrm{CO} 6 \mathrm{~cm}$ maser regions. We discuss the characteristics of the known $\mathrm{H}_{2} \mathrm{CO}$ masers and the association of $\mathrm{H}_{2} \mathrm{CO} 6 \mathrm{~cm}$ masers with very young regions of massive star formation. We also review the current ideas on the pumping mechanism for $\mathrm{H}_{2} \mathrm{CO} 6 \mathrm{~cm}$ masers.
\end{abstract}

Keywords. masers, stars: formation, ISM: molecules, HII regions, radio lines: ISM

\section{Introduction}

Formaldehyde $\left(\mathrm{H}_{2} \mathrm{CO}\right)$ was the first organic polyatomic molecule discovered in the interstellar medium. $\mathrm{H}_{2} \mathrm{CO}$ is an asymmetric top molecule, however the asymmetry is small; the moment of inertia for rotation of the molecule about the ' $c$ ' axis is slightly greater than the moment of inertia about the ' $b$ ' axis (Figure 1a). In the case of ortho- $\mathrm{H}_{2} \mathrm{CO}$ (i.e., when the nuclear spins of the hydrogen atoms are parallel), the small asymmetry causes splitting of the rotational states into closely spaced energy levels known as K-doublets (Figure 1b). Electric dipole transitions between low energy K-doublets $\left(\Delta K_{c}= \pm 1\right.$, $\Delta J=0$, Q-branch transitions, e.g., Townes \& Schawlow 1975) result in radio-frequency lines.

The first detection of $\mathrm{H}_{2} \mathrm{CO}$ was reported by Snyder et al. (1969). They found $\mathrm{H}_{2} \mathrm{CO}$ absorption in the $6 \mathrm{~cm}$ line $\left(\mathrm{J}_{\mathrm{K}_{\mathrm{a}} \mathrm{K}_{\mathrm{c}}}=1_{11}-1_{10} ; \nu_{o}=4829.6596 \mathrm{MHz}\right.$ for the $\mathrm{F}=2-2$ hyperfine component, Tucker et al. 1971), i.e., the K-doublet transition from the lowest ortho- $\mathrm{H}_{2} \mathrm{CO}$ energy levels (Figure $1 \mathrm{~b}$ ). Soon after the first detection of $\mathrm{H}_{2} \mathrm{CO}$, Palmer et al. (1969) discovered $\mathrm{H}_{2} \mathrm{CO} 6 \mathrm{~cm}$ absorption against the $2.7 \mathrm{~K}$ Cosmic Microwave Background $(\mathrm{CMB})$, implying an excitation temperature $\mathrm{T}_{e x}<2.7 \mathrm{~K}$ for the $\mathrm{H}_{2} \mathrm{CO} 6 \mathrm{~cm}$ K-doublet in Galactic dark clouds. The detection of $\mathrm{H}_{2} \mathrm{CO}$ absorption against the $\mathrm{CMB}$ (the so called anomalous absorption of $\mathrm{H}_{2} \mathrm{CO}$ ) required an anti-inversion (cooling) mechanism that was promptly recognized to be caused by $\mathrm{H}_{2}$ collisions (Townes \& Cheung 1969, Garrison et al. 1975, Evans et al. 1975a, Green et al. 1978).

Almost four decades after its discovery, the $\mathrm{H}_{2} \mathrm{CO} 6 \mathrm{~cm}$ line has been detected toward hundreds of regions in the Galaxy. $\mathrm{H}_{2} \mathrm{CO}$ has been observed in absorption against the CMB, Galactic and extragalactic radio continuum sources (e.g., Rodríguez et al. 2006, Young et al. 2004, Sewiło et al. 2004b, Watson et al. 2003, Araya et al. 2002, Downes et al. 1980). In sharp contrast to the ubiquitous $\mathrm{H}_{2} \mathrm{CO} 6 \mathrm{~cm}$ absorption line, $\mathrm{H}_{2} \mathrm{CO} 6 \mathrm{~cm}$ emission is an extremely rare phenomenon: $\mathrm{H}_{2} \mathrm{CO} 6 \mathrm{~cm}$ emission has been confirmed as megamaser emission only toward four extragalactic objects (Araya et al. 2004a, Baan private communication), found as thermal emission only toward the Orion BN/KL region 

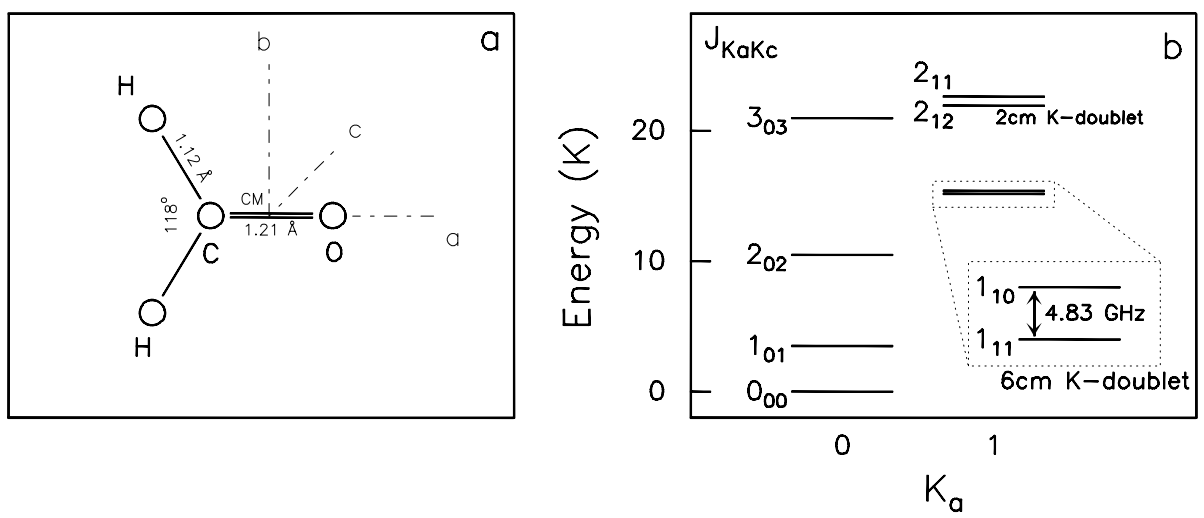

Figure 1. Left: Geometry of the $\mathrm{H}_{2} \mathrm{CO}$ molecule (Townes \& Schawlow 1975; for aesthetic reasons we show a 'left-handed' a-b-c coordinate axis). Right: $\mathrm{H}_{2} \mathrm{CO}$ energy level diagram of states with $\mathrm{E}\left(\mathrm{J}_{K_{a} K_{c}}\right)<30 \mathrm{~K}$ (Jaruschewski et al. 1986).

(Zuckerman et al. 1975, see also Araya et al. 2006b)†, and only seven Galactic maser regions have been reported (Forster et al. 1980, Whiteoak \& Gardner 1983, Pratap et al. 1994, Araya et al. 2005, 2006a, 2007 in prep.). In this article we review the field of $\mathrm{H}_{2} \mathrm{CO}$ $6 \mathrm{~cm}$ masers: the characteristics of the known masers, the astrophysical environments where the masers are found, and the current ideas on the excitation of $\mathrm{H}_{2} \mathrm{CO} 6 \mathrm{~cm}$ masers.

\section{2. $\mathrm{H}_{2} \mathrm{CO} 6 \mathrm{~cm}$ maser surveys}

The first $\mathrm{H}_{2} \mathrm{CO}$ maser region detected was NGC 7538. Using the Effelsberg $100 \mathrm{~m}$ telescope, Downes \& Wilson (1974) detected a double peak emission profile superimposed with an absorption feature (see Figure 2 for a recent spectrum of the maser). Aperture synthesis observations by Forster et al. (1980) and Rots et al. (1981) demonstrated the maser nature of the $\mathrm{H}_{2} \mathrm{CO} 6 \mathrm{~cm}$ emission. Approximately 10 years after the detection of $\mathrm{H}_{2} \mathrm{CO}$ emission in NGC 7538, Whiteoak \& Gardner (1983) using the VLA detected maser emission from five locations in Sgr B2. Mehringer et al. (1994) conducted further VLA observations $\left(\theta_{\text {syn }} \sim 1^{\prime \prime}\right)$ of the region and detected four more $\mathrm{H}_{2} \mathrm{CO}$ maser sites, resulting in a total of nine $\mathrm{H}_{2} \mathrm{CO} 6 \mathrm{~cm}$ maser sites in $\mathrm{Sgr}$ B2.

Since the detections by Downes \& Wilson (1974) and Whiteoak \& Gardner (1983), there have been six surveys specifically focused on the search for $\mathrm{H}_{2} \mathrm{CO}$ masers (see Table 1). Given the ubiquitous $\mathrm{H}_{2} \mathrm{CO} 6 \mathrm{~cm}$ absorption in molecular clouds (e.g., Watson et al. 2003) and the weak intensity of the known $\mathrm{H}_{2} \mathrm{CO} 6 \mathrm{~cm}$ masers (see $\S 3$ ), surveys for $\mathrm{H}_{2} \mathrm{CO}$ maser emission have been conducted using large single dish radio telescopes and interferometers to detect weak lines $(\sim 100 \mathrm{mJy})$ and to avoid confusion due to $\mathrm{H}_{2} \mathrm{CO}$ absorption.

The survey by Forster et al. (1985) focused on $\mathrm{OH}$ maser sources, including not only massive star forming regions but also OH maser stars. The survey yielded no new detections. Pratap et al. (1994) and Mehringer et al. (1995) conducted observations of active regions of massive star formation known to harbor ultra-compact H II regions as well as

$\dagger \mathrm{H}_{2} \mathrm{CO} 6 \mathrm{~cm}$ thermal emission was also reported toward comets Halley and Machholz (1988j) (Snyder et al. 1989, 1990; see however Bockelée-Morvan \& Crovisier 1992). Emission of the $2 \mathrm{~cm}$ K-doublet is also rare (Martín-Pintado et al. 1985, Johnston et al. 1984, Loren et al. 1983, Wilson et al. 1982, Evans et al. 1975b), and maser emission from the $2 \mathrm{~cm}$ transition has not been observed. 
Table 1. $\mathrm{H}_{2} \mathrm{CO} 6 \mathrm{~cm}$ Galactic masers

\begin{tabular}{|c|c|c|c|c|}
\hline Reference & Sample & Telescope & Detections & Selection Criteria \\
\hline Downes \& Wilson (1974) & 1 & Effelsberg & NGC $7538^{*}$ & NGC 7538 \\
\hline Whiteoak \& Gardner (1983) & 1 & VLA & Sgr B2* & Sgr B2 \\
\hline Forster et al. (1985) & 19 & WSRT & - & OH Maser Sources \\
\hline Pratap et al. (1994) & 7 & VLA & G29.96-0.02* & UCHII Regions \\
\hline Mehringer et al. (1995) & 22 & VLA & - & MSFR \\
\hline Araya et al. (2004b) & 15 & Arecibo & IRAS $18566+0408$ & Weak Cont. MSFR \\
\hline Araya et al. (2007a) & 58 & GBT/VLA & G23.71-0.20* & MSFR, $\mathrm{H}_{2} \mathrm{CO}$ Spectra \\
\hline Araya et al. (2007 in prep.) & 14 & VLA & $\begin{array}{l}\text { G23.01-0.41 \& } \\
\text { G25.83-0.18 }\end{array}$ & MSFR, $\mathrm{H}_{2} \mathrm{CO}$ Spectra \\
\hline
\end{tabular}

* Sources that have been observed with MERLIN and/or the VLBA (see §3).

maser emission from a variety of molecules. Out of 29 sources observed with the VLA in these surveys, only G29.96-0.02 was found to harbor $\mathrm{H}_{2} \mathrm{CO}$ maser emission.

Recently, Araya and collaborators conducted three surveys exploring different search strategies: 1 . they observed regions of weak radio continuum to reduce confusion due to $\mathrm{H}_{2} \mathrm{CO}$ absorption and focused on massive star forming regions thought to be in an evolutionary stage prior to the ultra-compact H II phase (Arecibo and GBT; Araya et al. 2004b, 2007a), 2. they observed massive star forming regions independently of the radio continuum to search for strong and potentially variable masers (GBT; Araya et al. 2007a), and 3. they conducted VLA observations of sources that had been previously observed with the GBT or Arecibo and that showed complex absorption line profiles consistent with $\mathrm{H}_{2} \mathrm{CO}$ emission blended with absorption (Araya et al. 2007a, 2007 in prep.). The three surveys resulted in detection of four new maser regions: IRAS $18566+0408$ (Araya et al. 2004b, 2005), G23.71-0.20 (Araya et al. 2006a, 2007a), G23.01-0.41 and G25.83-0.18 (Araya et al. 2007 in prep.).

\section{Physical properties}

$\mathrm{H}_{2} \mathrm{CO}$ masers are weaker in comparison with most $\mathrm{OH}, \mathrm{H}_{2} \mathrm{O}$, and $\mathrm{CH}_{3} \mathrm{OH}$ masers; the flux density range of the known $\mathrm{H}_{2} \mathrm{CO}$ masers is between $10 \mathrm{mJy}$ (for the maser in IRAS 18566+0408; see the poster contribution by Araya et al. in these proceedings) and $\sim 2 \mathrm{Jy}$ (for the brightest masers in Sgr B2 and NGC 7538; Hoffman et al. 2007, Araya et al. 2007a), while most masers have flux densities of the order of $\sim 100 \mathrm{mJy}$. Two maser regions have been observed at $\sim 50$ mas angular resolution with MERLIN: NGC 7538 (Hoffman et al. 2003), and G23.71-0.20 (Araya et al. 2007 in prep). MERLIN observations recover most ( $\gtrsim 70 \%)$ of the flux density detected at lower angular resolutions, and the masers are unresolved or barely resolved. Araya et al. (2007 in prep.) find a brightness temperature $\gtrsim 10^{5} \mathrm{~K}$ for the maser in G23.71-0.20.

Three sources have been observed at $\sim 10$ mas angular resolution with the VLBA: NGC 7538, G29.96-0.02, and Sgr B2. Hoffman et al. (2003) report brightness temperatures between $10^{6}$ and $10^{8} \mathrm{~K}$ for the masers in G29.96-0.02 and NGC 7538. Two of the nine maser components in Sgr B2 were observed with the VLBA by Hoffman et al. (2007). They also found brightness temperatures in the $10^{8} \mathrm{~K}$ range. In general, only a fraction of the flux density is recovered with the VLBA, and as in the case of other astrophysical masers, the lines are narrower in the VLBA observations compared with VLA or single dish observations. Hoffman et al. (2007) discuss these results in the context of a core-halo model, where the maser brightness distribution is the result of the superposition of two Gaussian components, one compact $(\sim 10$ mas) saturated component that is 

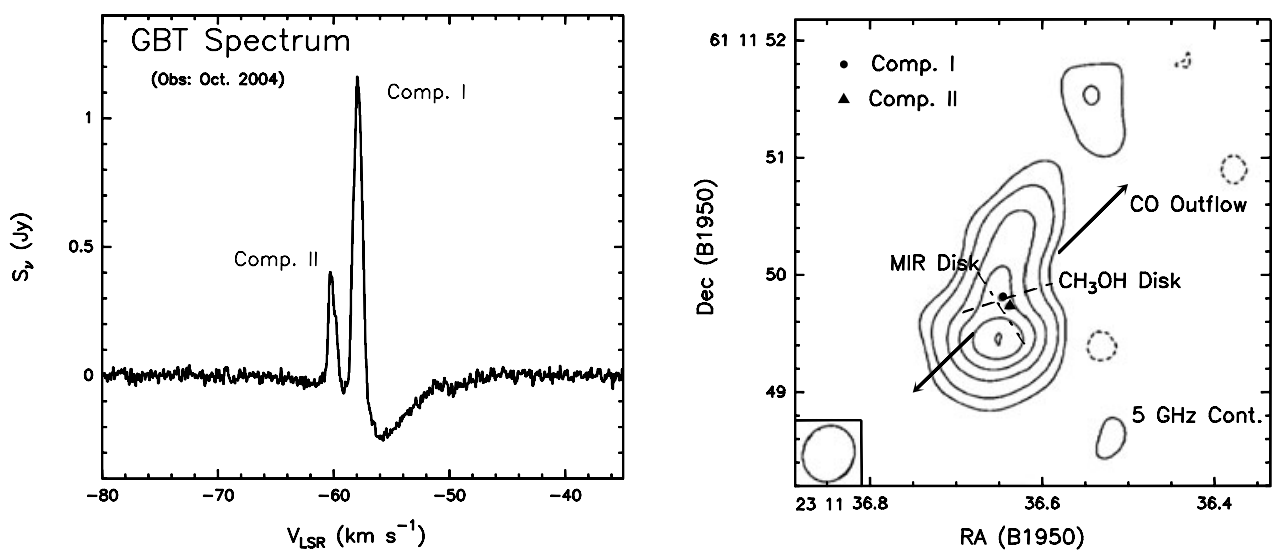

Figure 2. Left: $\mathrm{H}_{2} \mathrm{CO} 6 \mathrm{~cm}$ spectrum of NGC 7538 IRS1 (Araya et al. 2007a). Two maser components are blended with an $\mathrm{H}_{2} \mathrm{CO}$ absorption line. Right: Location of the two $\mathrm{H}_{2} \mathrm{CO}$ maser components (dot and triangle) superimposed on the $6 \mathrm{~cm}$ radio continuum in NGC 7538 IRS1 (Rots et al. 1981). Re-reduction of the Rots et al. (1981) data using a more accurate position of the phase calibrator results in $\mathrm{H}_{2} \mathrm{CO}$ maser positions that are consistent with the VLBA and MERLIN values reported by Hoffman et al. (2003). The direction of the CO (2-1) outflow is indicated by arrows (Davis et al. 1998), and two possible orientations of a circumstellar disk in the region are shown with dot-dashed (MIR Disk, De Buizer \& Minier 2005) and dashed $\left(\mathrm{CH}_{3} \mathrm{OH}\right.$ Disk, Pestalozzi et al. 2004) lines.

detected with the VLBA $\left(\mathrm{T}_{b} \sim 10^{8} \mathrm{~K}\right)$, and one extended and unsaturated halo that is resolved out by the VLBA observations $\left(\mathrm{T}_{b} \sim 10^{5} \mathrm{~K}\right)$. Based on the VLBA and MERLIN results, the projected physical size of the masers is between 30 and $\sim 200 \mathrm{AU}$, while the maser gains range between -6 and -12 ; the emission is unpolarized within the current sensitivity limits (Hoffman et al. 2003, 2007).

\section{Line profiles and velocity gradients}

With the exception of Sgr B2 where nine $\mathrm{H}_{2} \mathrm{CO}$ maser spots have been found (some of them showing multiple-peaked and broad line profiles, e.g., Mehringer et al. 1994), the $\mathrm{H}_{2} \mathrm{CO}$ maser line profiles are relatively simple. A peculiar characteristic of the known $\mathrm{H}_{2} \mathrm{CO}$ maser regions is that double peaked profiles are very common; double peaked profiles have been detected in NGC 7538 (e.g., Figure 2), G29.96-0.02 (Pratap et al. 1994, Hoffman et al. 2003), IRAS 18566+0408 (Araya et al. 2007c), G25.83-0.18 (Figure 3), and possibly toward G23.01-0.41 (Araya et al. 2007 in prep.). In all of these cases the velocity separation of the maser components is less than $3 \mathrm{~km} \mathrm{~s}^{-1}$, and the components are (in most cases) spatially coincident in VLA observations. The double peaked profiles are unlikely caused by the hyperfine structure of the $6 \mathrm{~cm} \mathrm{H}_{2} \mathrm{CO}$ transition. Recent high spectral resolution $\left(0.1 \mathrm{~km} \mathrm{~s}^{-1}\right.$ channel width) VLA observations of the masers in G23.01-0.41 and G25.83-0.18 (Araya et al. 2007 in prep.) show very narrow maser components (FWHM $\sim 0.3 \mathrm{~km} \mathrm{~s}^{-1}$ ), possibly due to the line narrowing effect of unsaturated masers.

In the case of the $\mathrm{H}_{2} \mathrm{CO}$ masers in NGC 7538, the components are oriented in a NESW direction, with a projected separation of 79 mas (240 AU). VLBA observations of the red shifted component show a $1900 \mathrm{~km} \mathrm{~s}^{-1} \mathrm{pc}^{-1}$ velocity gradient also in a NE-SW orientation (see figures 4 and 5 of Hoffman et al. 2003). 


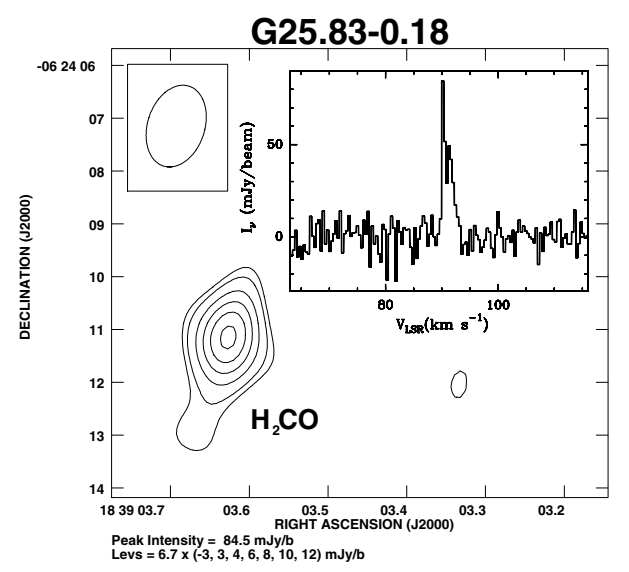

Figure 3. $\mathrm{H}_{2} \mathrm{CO} 6 \mathrm{~cm}$ maser emission in G25.83-0.18 detected with the VLA by Araya et al. (2007 in prep.). Excluding the masers in Sgr B2, five out of six sources show double peak profiles.

\section{Variability}

Prior to the recent work by Araya et al. (2007c), variability of $\mathrm{H}_{2} \mathrm{CO}$ masers had been observed only in some of the Sgr B2 masers and in the NGC 7538 masers; only long timescale variability (>1yr) had been reported (e.g., Mehringer et al. 1994, Hoffman et al. 2003). In the case of the variability of the $\mathrm{H}_{2} \mathrm{CO}$ masers in NGC 7538 and based on the similar variability-rate of the two maser components (Figure 4 left panel), Araya et al. (2007a) suggested that the variability of the masers may be caused by a perturbation that took $\sim 14 \mathrm{yr} \dagger$ to reach Comp. II after having reached Comp. I. If that were the case, an increase in the rate-of-change of the intensity of Comp. II would be expected after the year 2009. The variability of the two maser components could be related to the precessing jet reported by Kraus et al. (2006).

Araya et al. (2007c) have recently found a new type of $\mathrm{H}_{2} \mathrm{CO}$ maser variability, namely, short term flares. Using Arecibo, VLA, and GBT data, Araya et al. (2007c) reported occurrence of an outburst of the $\mathrm{H}_{2} \mathrm{CO} 6 \mathrm{~cm}$ maser in IRAS $18566+0408$; the maser flare lasted for less than three months and decayed to the pre-flare intensity within a month. The $\mathrm{H}_{2} \mathrm{CO}$ maser in IRAS $18566+0408$ has a double peak profile. Both components varied by approximately the same factor and in the same time period; no change in the line widths and peak velocities was detected. Araya et al. (2007c) discussed the implications of the flare on the possible excitation mechanism of the maser, and concluded that if the flare were due to a maser gain change, then (independently of the saturation state of the maser) the pumping mechanism is likely radiative; whereas if the maser is unsaturated, then a change in the background $6 \mathrm{~cm}$ radio continuum might have been amplified by the maser gas (independently on the maser pumping mechanism).

A monitoring program of the maser with Arecibo has recently resulted in the detection of a second $\mathrm{H}_{2} \mathrm{CO}$ maser burst in IRAS $18566+0408$ (Figure 4, right panel); showing that the flares are recurrent in this source. Araya and collaborators are also monitoring the $\mathrm{CH}_{3} \mathrm{OH}$ 6.7 GHz masers in the region, and found that one of the $\mathrm{CH}_{3} \mathrm{OH}$ maser components showed the same outburst as the $\mathrm{H}_{2} \mathrm{CO} 6 \mathrm{~cm}$ maser (Figure 4, right panel). The $\mathrm{CH}_{3} \mathrm{OH}$ maser peak that showed the flare does not correspond in velocity to the $\mathrm{H}_{2} \mathrm{CO}$ maser, hence the masers originate in different regions. It is possible that both masers are unsaturated and that a change in the background radio continuum was amplified by the

$\dagger$ Curiously, the period of the long-term variability of $\mathrm{H}_{2} \mathrm{O}$ masers in the region reported by Lekht et al. (2004) is $\sim 13 \mathrm{yr}$. 

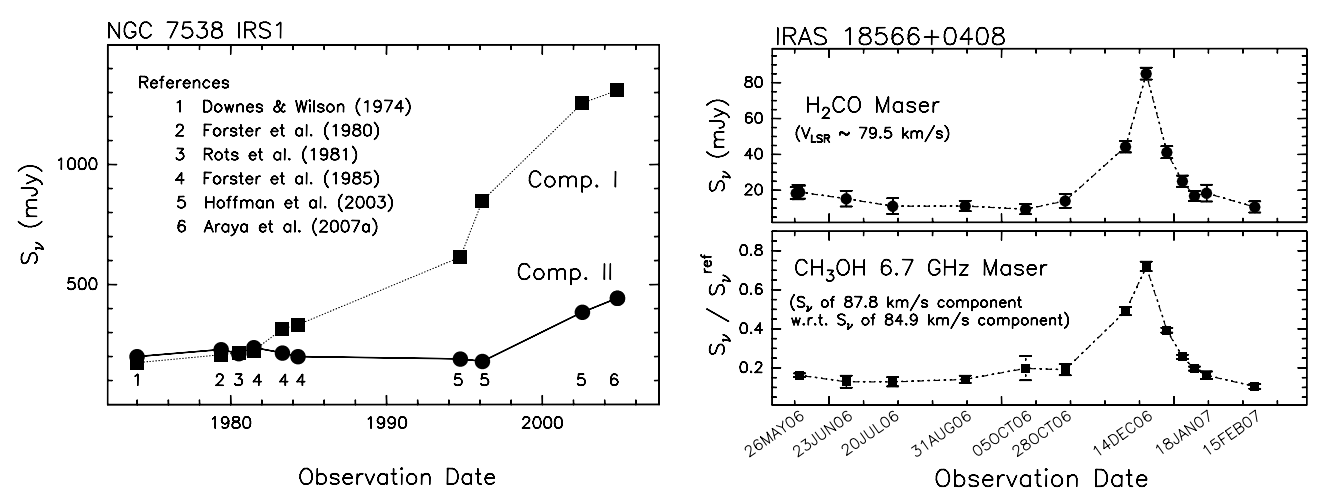

Figure 4. Left: Long term variability of the $\mathrm{H}_{2} \mathrm{CO}$ masers in NGC 7538. The similar intensity rate-of-change of the two maser components after the onset of the variability lead Araya et al. (2007a) to propose that the variability of both components may have a common origin. Right: Arecibo light-curve of the second short-term $\mathrm{H}_{2} \mathrm{CO}$ maser flare detected (Araya et al. 2007 in prep). Araya and collaborators are also monitoring with Arecibo the $\mathrm{CH}_{3} \mathrm{OH} 6.7 \mathrm{GHz}$ masers; one of the $\mathrm{CH}_{3} \mathrm{OH}$ maser components showed the same outburst as the $\mathrm{H}_{2} \mathrm{CO} 6 \mathrm{~cm}$ maser (see the poster contribution by Araya and collaborators in these proceedings).

$\mathrm{CH}_{3} \mathrm{OH}$ and $\mathrm{H}_{2} \mathrm{CO}$ masers (see poster contribution by Araya and collaborators in these proceedings).

Variability of some of the $\mathrm{H}_{2} \mathrm{CO}$ masers in Sgr B2 has also been found (Mehringer et al. 1994, Hoffman et al. 2007); however the available data are insufficient to establish whether the masers show long term variability or maser flares as in IRAS $18566+0408$.

\section{Astrophysical environments: $\mathrm{H}_{2} \mathrm{CO}$ masers pinpointing disk candidates around young massive stars}

Motivated by the detection of $\mathrm{H}_{2} \mathrm{CO}$ maser emission toward NGC 7538 and Sgr B2 (both massive star forming regions) the subsequent surveys for $\mathrm{H}_{2} \mathrm{CO}$ maser emission have been conducted mainly toward regions of massive star formation (Table 1). However, $\mathrm{H}_{2} \mathrm{CO} 6 \mathrm{~cm}$ observations have also been carried out toward a number of non-massive star forming regions and no new maser has been reported (e.g., Araya et al. 2006b, 2003; Rodríguez et al. 2006; Young et al. 2004). Thus, $\mathrm{H}_{2} \mathrm{CO} 6 \mathrm{~cm}$ masers appear to be exclusively associated with massive star formation.

Except for some of the $\mathrm{H}_{2} \mathrm{CO}$ masers in Sgr B2, $\mathrm{H}_{2} \mathrm{CO}$ masers are mostly found along line-of-sights devoid of strong compact radio continuum (though continuum regions may be found nearby, e.g., Pratap et al. 1994); they are located close to CLASS II $\mathrm{CH}_{3} \mathrm{OH}$ and $\mathrm{H}_{2} \mathrm{O}$ masers (in many cases coincident within a synthesized beam), deeply embedded infrared sources and/or other evidence of massive star formation such as hyper-compact H II regions and hot molecular cores (e.g., Araya et al. 2007b, 2006a, 2005; Hoffman et al. 2007, 2003; Pratap et al. 1994). Thus, $\mathrm{H}_{2}$ CO masers appear to trace young massive stellar objects before the onset of a bright ultra-compact HII region. Moreover, in the case of three of the $\mathrm{H}_{2} \mathrm{CO}$ maser sources that have been studied in detail, there is some evidence for an association between $\mathrm{H}_{2} \mathrm{CO}$ maser emission and circumstellar disks:

- G29.96-0.02 is a massive star forming region that harbors an ultra-compact HII region and a hot molecular core (e.g., Cesaroni et al. 1994). $\mathrm{The}_{2} \mathrm{CO}$ maser is coincident with the hot molecular core (Pratap et al. 1994). Hot molecular cores are believed to be an evolutionary phase prior to the formation of an ultra-compact H II region (e.g., 


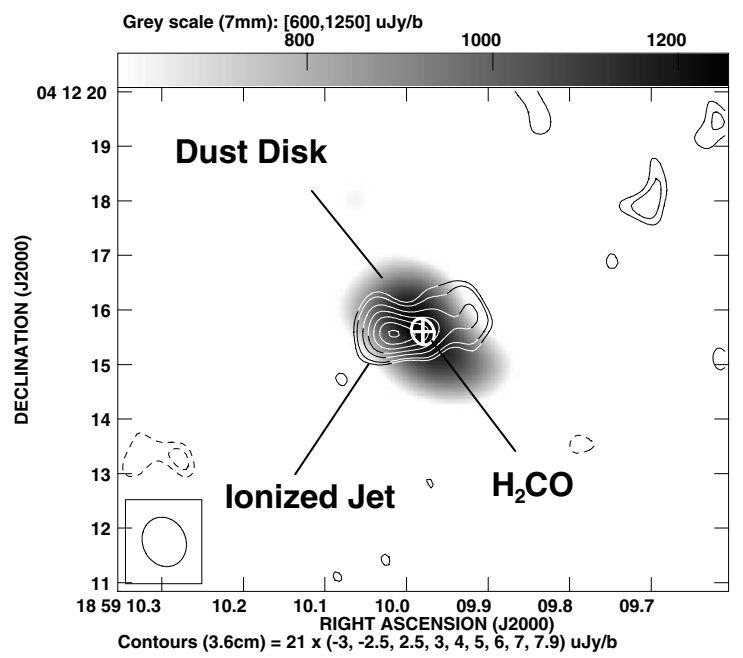

Figure 5. Location of the $\mathrm{H}_{2} \mathrm{CO} 6 \mathrm{~cm}$ maser in IRAS $18566+0408$ with respect to $7 \mathrm{~mm}$ (gray scale) and $3.6 \mathrm{~cm}$ (contours) continuum detected with the VLA. Araya et al. (2007b) concluded that the $3.6 \mathrm{~cm}$ emission is tracing an ionized jet whereas the $7 \mathrm{~mm}$ emission is dominated by dust emission from a possible massive circumstellar disk (torus). The $\mathrm{H}_{2} \mathrm{CO}$ maser is coincident with the massive disk candidate.

Churchwell 2002), thus the $\mathrm{H}_{2} \mathrm{CO}$ maser pinpoints a very young region of massive star formation. Olmi et al. (2003) report evidence of infall and a massive rotating disk in the hot molecular core. Thus, the $\mathrm{H}_{2} \mathrm{CO} 6 \mathrm{~cm}$ emission may be associated with a disk around a massive young stellar object. However, the assumption of a single massive disk might be over-simplistic given the complex sub-mm morphology found by Beuther et al. (2007).

- NGC 7538 IRS1 is a massive star forming region which harbors a hyper-compact H II region (e.g., Sewiło et al. 2004a), and a CO outflow centered at the NGC 7538 IRS1 position (Figure 2, right panel). NGC 7538 IRS1 is one of the few sources where a circumstellar disk around a massive (proto)star has been reported. However, the orientation of the disk is controversial. Based on $\mathrm{CH}_{3} \mathrm{OH}$ maser data by Minier et al. $(1998,2000,2001)$, Pestalozzi et al. (2004) report a possible Keplerian disk oriented $\sim$ SE-NW. However, based on mid-IR observations, De Buizer \& Minier (2005) considered that the $\mathrm{CH}_{3} \mathrm{OH}$ masers are tracing the outflow and that the disk is oriented in a NE-SW direction (i.e., perpendicular to the $\mathrm{CO}$ outflow, see Figure 2, right panel). As mentioned in $\S 4$, the two $\mathrm{H}_{2} \mathrm{CO}$ maser spots are oriented NE-SW, and the velocity gradient of Comp. I is also in the NE-SW direction, i.e., perpendicular to the $\mathrm{CO}$ outflow and parallel to the MIR disk. The $\mathrm{H}_{2} \mathrm{CO} 6 \mathrm{~cm}$ masers appear to trace material very close to (within $1000 \mathrm{AU}$ ) or directly associated with a circumstellar disk.

- IRAS 18566+0408 was classified by Zhang (2005) as a massive circumstellar disk candidate. Based on high sensitivity and angular resolution $6,3.6,1.3$, and $0.7 \mathrm{~cm}$ VLA continuum observations, Araya et al. (2007b) recently found supporting evidence for the presence of a massive circumstellar disk in IRAS 18566+0408 (see Figure 5). The massive disk (torus) is traced by $7 \mathrm{~mm}$ dust emission and has an elongation almost perpendicular to an ionized jet traced by $\mathrm{cm}$ radio continuum. The $\mathrm{H}_{2} \mathrm{CO}$ maser is coincident with the center of the massive disk candidate. 


\section{The pumping mechanism of $\mathrm{H}_{2} \mathrm{CO} 6 \mathrm{~cm}$ masers}

Although the number of $\mathrm{H}_{2} \mathrm{CO} 6 \mathrm{~cm}$ maser sources is still small, in the past few years significant progress in the characterization of $\mathrm{H}_{2} \mathrm{CO}$ masers and their environments has been made; however, a theoretical understanding of the excitation mechanism of $\mathrm{H}_{2} \mathrm{CO}$ masers is still lacking. Even before the detection of the first $\mathrm{H}_{2} \mathrm{CO}$ maser, several authors mentioned and/or discussed possible excitation mechanisms that would result in maser emission of the $6 \mathrm{~cm}$ line; including collisional excitation with $\mathrm{H}_{2}$ molecules and electrons (Thaddeus 1972; see also Fig. 14 of Evans et al. 1975a), and infrared pumping (Litvak 1970). However, only Boland \& de Jong (1981) developed a specific model to explain one of the known $\mathrm{H}_{2} \mathrm{CO}$ masers (NGC 7538). This model is based on inversion via background radio continuum radiation; however, the model appears to be incapable of explaining most of the known $\mathrm{H}_{2} \mathrm{CO}$ masers (Araya et al. 2007b, Hoffman et al. 2007, 2003, Pratap et al. 1994, Mehringer et al. 1994; see however Pratap et al. 1992).

Besides inversion by radio continuum, other proposed excitation mechanisms appear to be possible: 1. Hoffman et al. $(2007,2003)$ and Araya et al. (2005) find indications that the masers could be collisionally excited in shocked regions (see also Martín-Pintado et al. 1999), 2. some $\mathrm{H}_{2} \mathrm{CO}$ masers are found close to deeply embedded infrared objects and thus infrared pumping could be possible (Araya et al. 2006a), and 3. $\mathrm{H}_{2} \mathrm{CO}$ masers are located close to very young massive stellar objects where a high ionization fraction is expected, and thus electron collision may play a role in the pumping.

Araya et al. (2007 in prep.) explore excitation of $\mathrm{H}_{2} \mathrm{CO}$ masers via $\mathrm{H}_{2}$ and electron collisions and have found that collision with electrons can indeed produce an inversion of the $6 \mathrm{~cm} \mathrm{~K}$-doublet (see also Thaddeus 1972). However, preliminary results by Araya et al. (2007 in prep.) appear to require long path lengths ( $\sim$ parsec scales) to reproduce the brightness temperature of the known $\mathrm{H}_{2} \mathrm{CO}$ masers. Parsec-scale path lengths of coherent velocity and homogeneous physical conditions in massive star forming regions are unlikely. However pumping of $\mathrm{H}_{2} \mathrm{CO}$ by electron collisions appears to be a promising mechanism to explain extragalactic megamasers (Araya et al. 2007 in prep., see also Araya et al. 2004a; Baan \& Haschick 1995). Araya et al. (2007 in prep.) also find that including radiation trapping, the $6 \mathrm{~cm}$ K-doublet may be inverted at a molecular density of $\sim 10^{6} \mathrm{~cm}^{-3}$ (i.e., in the transition between anomalous $\mathrm{H}_{2} \mathrm{CO}$ absorption and thermalization). However, the model depends on accurate $\mathrm{H}_{2}$ (ortho/para) $-\mathrm{H}_{2} \mathrm{CO}$ collision rates which are not available at present (e.g., Green 1991; see also Hoffman et al. 2003).

\section{Why are $\mathrm{H}_{2} \mathrm{CO}$ masers so rare?}

In spite of a number of surveys specifically focused on the search for $\mathrm{H}_{2} \mathrm{CO}$ masers and hundreds of sources for which $\mathrm{H}_{2} \mathrm{CO} 6 \mathrm{~cm}$ absorption studies have been conducted (e.g., Table 1, Araya et al. 2002, Watson et al. 2003, Sewiło et al. 2004), $\mathrm{H}_{2} \mathrm{CO}$ maser emission has been detected only toward seven regions (and in a total of 15 maser spots at $1^{\prime \prime}$ resolution). Thus, $\mathrm{H}_{2} \mathrm{CO}$ maser emission is indeed a rare phenomenon. Reformulating the ideas presented by Mehringer et al. (1995), Pratap et al. (1992), and Forster et al. (1985), $\mathrm{H}_{2} \mathrm{CO}$ masers may be uncommon because: 1 . they are weak in comparison with other astrophysical masers and/or are highly beamed: the brightest known $\mathrm{H}_{2} \mathrm{CO}$ maser is just $\sim 2 \mathrm{Jy} ; 2$. they occur at LSR velocities close to the systemic velocity of the star forming regions and thus the maser emission is highly attenuated by large optical depths of the $\mathrm{H}_{2} \mathrm{CO}$ absorption line: $\mathrm{H}_{2} \mathrm{CO}$ masers are typically found within $\sim 5 \mathrm{~km} \mathrm{~s}^{-1}$ from the systemic cloud velocity as traced by $\mathrm{H}_{2} \mathrm{CO}$ absorption (e.g., Figure 2, Araya et al. 2004b) and are not found at high velocities, indicating that $\mathrm{H}_{2} \mathrm{CO}$ masers are not associated with high velocity outflows; and 3. the physical conditions needed for the 
excitation of the masers are very specific, and thus, short-lived in massive star forming region environments. For example, the $\mathrm{H}_{2} \mathrm{CO}$ masers (and $\mathrm{H}$ II regions) in Sgr $\mathrm{B} 2$ are distributed in a N-S direction suggesting that star formation in Sgr B2 was triggered by a cloud collision event (see Sato et al. 2000) and thus the masers may be tracing an isochrone of the physical conditions during the massive star formation process (see Gardner et al. 1986).

\section{Summary}

Despite the ubiquitous presence of $\mathrm{H}_{2} \mathrm{CO} 6 \mathrm{~cm}$ absorption, there are only seven known $\mathrm{H}_{2} \mathrm{CO}$ maser regions in the Galaxy. Recent VLBA and MERLIN observations toward four sources show brightness temperatures between $10^{5}-10^{9} \mathrm{~K}$, and physical sizes between 30 $200 \mathrm{AU}$. At least two masers show long-term variability ( $>1$ year), and one maser source was recently shown to exhibit recurrent short-term ( $<3$ months) bursts. All known $\mathrm{H}_{2} \mathrm{CO}$ $6 \mathrm{~cm}$ masers are found in massive star forming regions, and in the case of the three sources that have been studied in detail (NGC 7538 IRS1, G29.96-0.02, and IRAS 18566+0408; excluding Sgr B2) the $\mathrm{H}_{2} \mathrm{CO}$ masers pinpoint the location of candidate disks around massive protostars. However, the $\mathrm{H}_{2} \mathrm{CO}$ maser mechanism has to be clarified before the masers can be used as an astrophysical probe.

\section{Acknowledgements}

The research on $\mathrm{H}_{2} \mathrm{CO} 6 \mathrm{~cm}$ masers by the author (E.A.) has greatly benefited from collaboration with H. Linz, S. Kurtz, W. Baan, M. Sewiło, E. Churchwell, L. Olmi, C. Watson, I. Hoffman, L. F. Rodríguez, G. Garay, \& P. Palmer. E. A. is supported by a NRAO predoctoral fellowship. We thank H. Linz and I. Hoffman for comments that improved the manuscript, and H. Beuther for sharing information about the G29.96-0.02 small scale structure based on SMA sub-mm data prior to publication. E. A. thanks the IAU for a travel grant to attend this symposium.

\section{References}

Araya, E., Baan, W., \& Hofner, P. 2004a, ApJS 154, 541

Araya, E., Hofner, P., Churchwell, E., \& Kurtz, S. 2002, ApJS 138, 63

Araya, E., et al. 2007b, ApJ submitted

Araya, E., Hofner, P., Goldsmith, P., Slysh, S., \& Takano, S. 2003, ApJ 596, 556

Araya, E., Hofner, P., Goss, W. M., Kurtz, S., Linz, H., \& Olmi, L. 2006a, ApJ (Letters) 643, L33

Araya, E., Hofner, P., Goss, W. M., Linz, H., Kurtz, S., \& Olmi, L. 2007a, ApJS, 170, 152

Araya, E., Hofner, P., Kurtz, S., Linz, H., Olmi, L., Sewiło, M., Watson, C., \& Churchwell, E. 2005, ApJ 618, 339

Araya, E., Hofner, P., Linz, H., Sewiło, M., Watson, C., Churchwell, E., Olmi, L., \& Kurtz, S. 2004b, ApJS 154, 579

Araya, E., Hofner, P., Olmi, L., Kurtz, S., \& Linz, H. 2006b, AJ 132, 1851

Araya, E., Hofner, P., Sewiło, M., Linz, H., Kurtz, S., Olmi, L., Watson, C., \& Churchwell, E. 2007c, ApJ (Letters) 654, L95

Baan, W. A., \& Haschick, A. D. 1995, ApJ 454, 745

Beuther, H., Zhang, Q., Bergin, E. A., et al. 2007, A\&A, accepted (arXiv:0704.0518)

Bockelée-Morvan, D., \& Crovisier, J. 1992, A\&\&A 264, 282

Boland, W., \& de Jong, T. 1981, A\&A 98, 149

Cesaroni, R., Churchwell, E., Hofner, P., Walmsley, C. M., \& Kurtz, S. 1994, A\&\&A 288, 903

Churchwell, E. 2002, ARA\&A 40, 27

Davis, C. J., Moriarty-Schieven, G., Eislöffel, J., Hoare, M. G., \& Ray, T. P. 1998, AJ 115, 1118

De Buizer, J. M., \& Minier, V. 2005, ApJ (Letters) 628, L151

Downes, D., \& Wilson, T. L. 1974, ApJ (Letters) 191, L77 
Downes, D., Wilson, T. L., Bieging, J., \& Wink, J. 1980, A\&AS 40, 379

Evans, N. J. II, Zuckerman, B., Morris, G., \& Sato, T. 1975a, ApJ 196, 443

Evans, N. J. II, Zuckerman, B., Sato, T., \& Morris, G. 1975b, ApJ 199, 383

Forster, J. R., Goss, W. M., Gardner, F. F., \& Stewart, R. T. 1985, MNRAS 216, 35

Forster, J. R., Goss, W. M., Wilson, T. L., Downes, D., \& Dickel, H. R. 1980, $A \mathscr{E} A$ (Letters) 84, L1

Gardner, F. F., Whiteoak, J. B., \& Forster, J. R. 1986, MNRAS 218, 385

Garrison, B. J., Lester, W. A. Jr., Miller, W. H., \& Green, S. 1975, ApJ (Letters) 200, L175

Green, S. 1991, ApJS 76, 979

Green, S., Garrison, B. J., Lester, W. A. Jr., \& Miller, W. H. 1978, ApJS 37, 321

Hoffman, I. M., Goss, W. M., \& Palmer, P. 2007, ApJ 654, 971

Hoffman, I. M., Goss, W. M., Palmer, P., \& Richards, A. M. S. 2003, ApJ 598, 1061

Jaruschewski, S., Chandra, S., Varshalovich, D. A., \& Kegel, W. H. 1986, A\&AS 63, 307

Johnston, K. J., Henkel, C., \& Wilson, T. L. 1984, ApJ (Letters) 285, L85

Kraus, S., Balega, Y., Elitzur, M., Hofmann, K.-H., Preibisch, Th., Rosen, A., Schertl, D., Weigelt, G., \& Young, E. T. 2006, A\&\&A 455, 521

Lekht, E. E., Munitsyn, V. A., \& Tolmachev, A. M. 2004, Astron. Rep. 48, 200

Litvak, M. M. 1970, ApJ (Letters) 160, L133

Loren, R. B., Sandqvist, A., \& Wootten, A. 1983, ApJ 270, 620

Martín-Pintado, J., Gaume, R. A., Rodríguez-Fernández, N., de Vicente, P., \& Wilson, T. L. 1999, ApJ 519, 667

Martín-Pintado, J., Wilson, T. L., Gardner, F. F., \& Henkel, C. 1985, A\&\&A 142, 131

Mehringer, D. M., Goss, W. M., \& Palmer, P. 1994, ApJ 434, 237

Mehringer, D. M., Goss, W. M., \& Palmer, P. 1995, ApJ 452, 304

Minier, V., Booth, R., \& Conway, J. 1998, A\&A (Letters) 336, L5

Minier, V., Booth, R., \& Conway, J. 2000, A\&\&A 362, 1093

Minier, V., Conway, J., \& Booth, R. 2001, A\&A 369, 278

Olmi, L., Cesaroni, R., Hofner, P., Kurtz, S., Churchwell, E., \& Walmsley, C. M. 2003, A\&A 407, 225

Palmer, P., Zuckerman, B., Buhl, D., \& Snyder, L. E. 1969, ApJ (Letters) 156, L147

Pestalozzi, M. R., Elitzur, M., Conway, J. E., \& Booth, R. S. 2004, ApJ (Letters) 603, L113

Pratap, P., Menten, K. M., \& Snyder, L. E. 1994, ApJ (Letters) 430, L129

Pratap, P., Snyder, L. E., \& Batrla, W. 1992, ApJ 387, 241

Rodríguez, M. I., Allen, R. J., Loinard, L., \& Wiklind, T. 2006, ApJ 652, 1230

Rots, A. H., Dickel, H. R., Forster, J. R., \& Goss, W. M. 1981, ApJ (Letters) 245, L15

Sato, F., Hasegawa, T., Whiteoak, J. B., \& Miyawaki, R. 2000, ApJ 535, 857

Sewiło, M., Churchwell, E., Kurtz, S., Goss, W. M., \& Hofner, P. 2004a, ApJ 605, 285

Sewiło, M., Watson, C., Araya, E., Churchwell, E., Hofner, P., \& Kurtz, S. 2004b, ApJS 154, 553

Snyder, L. E., Buhl, D., Zuckerman, B., \& Palmer, P. 1969, Phys. Rev. Lett. 22, 679

Snyder, L. E., Palmer, P., \& de Pater, I. 1990, ICARUS 86, 289

Snyder, L. E., Palmer, P., \& de Pater, I. 1989, AJ 97, 246

Thaddeus, P. 1972, ApJ 173, 317

Townes, C. H., \& Cheung, C. 1969, ApJ (Letters) 157, L103

Townes, C. H., \& Schawlow, A. L. 1975, Microwave Spectroscopy (New York: Dover)

Tucker, K. D., Tomasevich, G. R., \& Thaddeus, P. 1971, ApJ 169, 429

Watson, C., Araya, E., Sewiło, M., Churchwell, E., Hofner, P., \& Kurtz, S. 2003, ApJ 587, 714

Whiteoak, J. B., \& Gardner, F. F. 1983, MNRAS 205, 27

Wilson, T. L., Martín-Pintado, J., Gardner, F. F., \& Henkel, C. 1982, A\& A (Letters) 107, L10

Young, K. E., Lee, J. E., Evans, N. J., II, Goldsmith, P. F., \& Doty, S. D. 2004, ApJ 614, 252

Zhang, Q. 2005, in: R. Cesaroni, M. Felli, E. Churchwell, \& M. Walmsley (eds.), Massive Star Birth: A Crossroads of Astrophysics (Cambridge: Cambridge Univ. Press), p. 135

Zuckerman, B., Palmer, P., \& Rickard, L. J. 1975, ApJ 197, 571 